\title{
Parámetros de Transferencia de Materia en el Secado de Frutas
}

\author{
Sebastião R. Ferreira y Antônio R. S. Costa \\ Universidad Federal de Río Grande del Norte, Departamento de Ingeniería Química, Av. Salgado \\ Filho 3000, 59078-970 Natal, RN-Brasil (e-mail: ferreira@eq.ufrn.br, seba@ufrnet.br)
}

\begin{abstract}
Resumen
Se desarrolló un modelo analítico para obtención de parámetros de transferencia de materia en el secado de frutas. El modelo considera la disminución del radio de las frutas durante el proceso de secado. Con el modelo matemático se predice simultánemente el coeficiente externo de transferencia de materia $k_{m}$ y la difusividad de agua en los frutos $D_{A B}$. Para probar el modelo se obtuvo en forma expermental datos de masa de agua $M_{A}$ en la fruta versus el tiempo de secado $t$, además del radio inicial $R_{0}$ y el radio al final del proceso $R_{f}$. Los valores obtenidos de $D_{A B}$ y $k_{m}$ son del mismo orden de magnitud de los calculados por un método numérico de la literatura. La metodología desarrollada, considerando disminución de volumen, puede ser empleada para evaluar parámetros de secado con exactitud razonable para cálculos en ingeniería.
\end{abstract}

Palabras clave: transferencia de materia, secado de frutas, modelado matemático, difusividad

\section{Mass Transfer Parameters in Fruit Drying}

\begin{abstract}
An analytical model was developed to determine mass transfer parameters during fruit drying. The model considers radius shrinkage during the drying process. With the mathematical model the external mass transfer coefficient $k_{m}$ and the water diffusivity $D_{A B}$ can be simultaneously evaluated. To test the model, experimental data of water mass $M_{A}$ versus drying time $t$, and the initial radius $R_{0}$ and the radius at the end of the process $R_{f}$ were determined. The values obtained for $D_{A B}$ and $k_{m}$ were of the same order of magnitude as those calculated by a numerical method from the literature. The proposed methodology, that considers volume shrinkage, can be used to estimate drying parameters with acceptable accuracy for engineering calculations.
\end{abstract}

Keywords: mass transfer, fruit drying, mathematical modeling, diffusivity, 


\section{INTRODUCCIÓN}

Para la obtención de modelos de secado generalmente son consideradas las ecuaciones de transferencia de materia, energía y cantidad de movimiento, surgiendo naturalmente parámetros que influyen en el secado, tales como el coeficiente externo de transferencia de materia entre una fruta y el aire de secado $\mathrm{k}_{\mathrm{m}}(\mathrm{m} / \mathrm{s})$, la difusividad de agua en una fruta $\mathrm{D}_{\mathrm{AB}}\left(\mathrm{m}^{2} / \mathrm{s}\right)$ y el número de $B i o t \mathrm{Bi}_{\mathrm{m}}(\operatorname{adim}$.). Uno de los modelos de secado más conocidos es el de Luikov (1966), que está basado en la termodinámica de los procesos irreversibles (Abalone et al., 2001; Luikov, 1966; Pandey et al., 1999; Wu e Irudayaraj, 1996). El modelo de Luikov es constituido por ecuaciones diferenciales parciales acopladas, en función de la temperatura, la humedad y, cuando existe un intenso secado, puede incluir la presión.

En algunas investigaciones están contemplados los modelos de secado de frutos (Lima, 1999) y también del secador (Karim y Hawlader, 2005a, b; Mabrouk et al., 2006). Los modelos para el fruto y secador están acoplados a través de los flujos de materia, energía y cantidad de movimiento en la interfase fruta/aire. Lima (1999) se dedicó especialmente al análisis del secado de banana; siendo parte de su investigación reproducida en un trabajo posterior (Lima et al., 2002). En el trabajo original (Lima, 1999) desarrolló modelos bidimensionales, analíticos y numéricos, para simular la difusión en sólidos y, en particular, ellos fueron empleados para describir la transferencia de materia y energía en el secado de banana. En su investigación se destaca el denominado modelo (III), que es un modelo con condición de contorno convectiva, fenómenos simultáneos de transferencia de humedad y disminución de volumen del fruto. En su modelo (III) el citado autor admitió, entre otras consideraciones, que:

a) El secado ocurre bajo condición de contorno convectiva, con humedad dependiente de la posición en el sólido esferoidal y del tiempo. Un esquema de un sólido esferoidal es presentado en la Fig.(1).

b) El Biot es admitido como variable durante el proceso de secado; el cual es definido por la Ec.(3).

c) La disminución de volumen es linealmente proporcional a la pérdida promedio de humedad del sólido.

Según Lima, el modelo (III) es el más realista de los analizados por él, dando resultados confiables para los coeficientes transporte; porque están incluidos más efectos físicos que inciden en la cinética de secado, comparado con otros modelos desarrollados por este autor (Lima, 1999; Lima et al., 2002). Por ejemplo, para aire a $60,2{ }^{\circ} \mathrm{C}$, humedad relativa de $19,9 \%$ y velocidad de $0,36 \mathrm{~m} / \mathrm{s}$, Lima et al. (2002) presentan $D_{A B}=7,2510^{-10} \mathrm{~m}^{2} / \mathrm{s}, \mathrm{k}_{\mathrm{m}}=22,3010^{-8} \mathrm{~m} / \mathrm{s}$ y Biot en el inicio y final del proceso, respectivamente, $\mathrm{Bi}_{\mathrm{LO}}=17,52$ y $\mathrm{Bi}_{\mathrm{Lf}}=11,14$. Como $\mathrm{D}_{\mathrm{AB}}$ y $\mathrm{k}_{\mathrm{m}}$ son considerados constantes y $\mathrm{Bi}_{\mathrm{L}}$ no, entonces al analizar la Ec.(3) se deduce que $B i_{L}$ se modifica debido a la disminución de $L$ del fruto en el secado. En realidad, $D_{A B}, k_{m} y L$ pueden modificarse en el proceso.

En la Fig.(1), que representa la mitad de la longitud de una banana, están destacadas las relaciones entre los sistemas de coordenadas cartesianas $(x, y, z)$ y esferoidal $(\mu, \phi, \omega)$. Se presentan el semieje menor y mayor, $L_{1}$ y $L_{2}$, respectivamente, y la longitud focal $L$ que es calculada por la siguiente ecuación (Lima, 1999):

$$
L=\sqrt{\left(L_{2}^{2}-L_{1}^{2}\right)}
$$

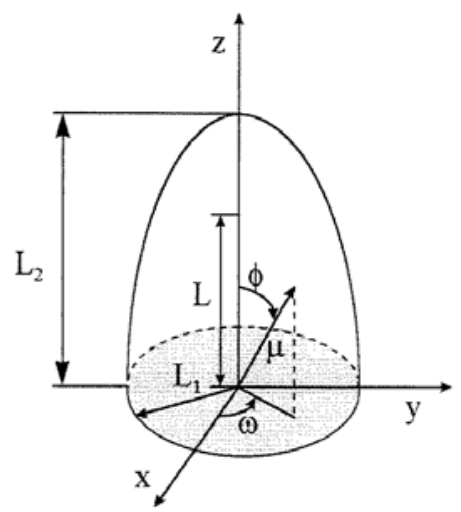

Fig. 1: Características de un sólido esferoidal (Lima, 1999; Teruel et al., 2001). 
El volumen de un sólido esferoidal, con $L_{1}<L_{2}$, es calculado por la ecuación (Lima, 1999):

$$
V=\frac{4}{3} \pi L_{1}^{2} L_{2}
$$

Cuando son cortadas las dos puntas de una banana se obtiene aproximadamente un cilindro finito y, en general, el valor de $L_{1}$ es aproximadamente igual al radio de una banana $R, y L$ es aproximadamente igual a la mitad de la longitud de una banana. El número de Biot de transferencia de materia $\mathrm{Bi}_{\llcorner}$, para un sólido esferoidal de longitud característica L, como el de la Fig.(1), es dado por (Lima, 1999):

$$
B i_{L}=\frac{k_{m} L}{D_{A B}}
$$

Para un cilindro finito existen dos números de Biot de transferencia de materia; uno para el radio R, dado por la Ec.(12), y otro para la longitud L, dado por la Ec.(3). Para un cilindro infinito hay sólo Biot para R.

Karim y Hawlader (2005a,b) desarrollaron un modelo de secado para un fruto, prediciendo su temperatura y humedad. Es admitido disminución del volumen del material y debido a esto $\mathrm{D}_{\mathrm{AB}}$ resultó dependiente del contenido acuoso y de la temperatura del fruto (Bird et al., 2002; Crank, 1976). La $D_{A B}$, obtenida por Karim y Hawlader, disminuye con el progreso del secado; debido a que la reducción del volumen produce cambios en la estructura del fruto, aumentando la resistencia a la difusión del agua, lo que disminuye $D_{A B}$. Mabrouk et al. (2006) desarrollaron un modelo numérico para predicción de la transferencia de materia y energía de productos granulares en un secador tipo túnel, de lecho fijo. Como la capa usada de producto (uva) era de pequeño espesor, admitieron que no existía gradiente de temperatura y humedad, y obtuvieron un modelo simplificado basado en una capa delgada.

Costa y Ferreira (2007), presentaron un modelo de secado, basándose en la ecuación de Fick para frutos cortados en forma de placa. Lo analizaron para $\mathrm{Bi} \rightarrow \infty$, esto es, sin resistencia externa a la transferencia de materia. Un modelo de este tipo es restrictivo, pues sirve sólo para averiguar como se comportaría el sistema cuando la resistencia externa a la transferencia de materia es despreciable. Si Biot fuese un parámetro libre, podría ser obtenido un valor diferente del impuesto, $\mathrm{Bi} \rightarrow \infty$, y quizás representase mejor la realidad del proceso de secado. Costa (2008), en colaboración con el co-autor de este artículo, presenta cálculos para cilindro infinito, ampliando su trabajo anterior (Costa y Ferreira, 2007).

Una de las dificultades en el modelado del secado de frutas es la obtención de parámetros de transporte, tales como $\mathrm{Bi}_{\mathrm{m}}, \mathrm{D}_{\mathrm{AB}}$ y $\mathrm{k}_{\mathrm{m}}$. Comúnmente para evaluarlos son empleadas correlaciones o son realizados experimentos por separado para calcularlos o son usados métodos numéricos, para analizar como ellos se comportan en función de variables del proceso. Por ejemplo, para resolver simultáneamente las ecuaciones de transferencia de energía y materia, Mariani et al. (2008) usaron correlaciones para evaluar el coeficiente convectivo de transferencia de calor, $\mathrm{h}_{\text {conv }}\left(\mathrm{Wm}^{-20} \mathrm{C}^{-1}\right)$, en vez de obtenerlo automáticamente del método numérico empleado. En general, esas correlaciones son válidas para una geometría dada, en función de los números de Nusselt, Prandtl y Reynolds (Bird et al., 2002).

El coeficiente $h_{\text {conv }}$ también puede ser evaluado de ensayo por separado, por ejemplo, con un cilindro de aluminio o de otro metal, de iguales dimensiones y en condiciones experimentales similares que una fruta dada, obteniendo $\mathrm{Bi}_{\mathrm{C}}=\mathrm{h}_{\text {conv }} \mathrm{R} / \mathrm{k}_{\mathrm{Al}} \rightarrow 0$, para el cilindro metálico. Como la conductividad térmica del aluminio, $\mathrm{k}_{\mathrm{Al}}$, es muy grande, en general, se cumple la condición $\mathrm{Bi}_{\mathrm{C}} \rightarrow 0$ y el $\mathrm{h}_{\text {conv }}$ puede evaluado de $\mathrm{T}$ versus $t$, en el cilindro de aluminio, con un modelo sencillo de transferencia de calor. El $h_{\text {conv }}$ obtenido para el cilindro metálico puede ser empleado para la fruta, en las condiciones ya mencionadas. Srikiatden y Roberts (2008) calcularon $\mathrm{h}_{\text {conv }}$ usando un procedimiento como el mencionado, empleándolo en un modelo de secado, considerando transferencias simultáneas de energía y materia, para evaluar $D_{A B}$ de agua en papas y zanahorias. Kaya et al. (2008) obtuvieron de experimentos el coeficiente local $\mathrm{h}_{\text {conv }}$, a través del flujo de calor en la superficie de un kiwi y calcularon $\mathrm{k}_{\mathrm{m}}$, usando la analogía de Chilton y Colburn, entre el espesor de capa límite de transferencia de calor y materia. Pero Lima (1999) evaluó $\mathrm{k}_{\mathrm{m}}$ 
de una banana en aire, a partir del ajuste de un modelo matemático, usando datos experimentales de secado, obtenidos por Queiroz (1994).

La masa de agua en una fruta a tiempo infinito $M_{A \infty}$, también puede ser evaluada en experimento por separado, para una temperatura dada, presión y concentración acuosa del ambiente. Partiendo de una masa inicial de agua en una fruta $M_{A 0}$, ella alcanza la masa $M_{A \infty}$ cuando entra en equilibrio con el medio, en las condiciones mencionadas. La masa $\mathrm{M}_{\mathrm{A} 0}$ también puede ser obtenida de un ensayo por separado, calculándose la cantidad inicial de agua en un alimento, a partir de un experimento en estufa. Kaya et al. (2008) obtuvieron experimentalmente la masa de equilibrio $M_{A e}$, para kiwi, a través de isotermas, empleando soluciones saturadas de sales y agua destilada, a $25,35,45,55$ y $65^{\circ} \mathrm{C}$, en función de la humedad relativa en el rango del 9,6 al $100 \%$. Evaluaron $M_{A 0}$ de kiwi, con un equipamiento de infrarrojo, que analiza concentración acuosa.

En esta investigación se propone un modelo para evaluar parámetros, tales como $\mathrm{Bi}_{\mathrm{m}}, \mathrm{D}_{\mathrm{AB}}$ y $\mathrm{k}_{\mathrm{m}}$. En la metodología desarrollada, algunos parámetros son mantenidos libres durante sus cálculos, por ejemplo, $\mathrm{Bi}_{\mathrm{m}}$ y $\mathrm{D}_{\mathrm{AB}}$, esto es, ellos son determinados automáticamente por un programa computacional de regresión no lineal y representan valores promedio para el secado. Además, son comparados los resultados obtenidos del modelo propuesto y los calculados por Lima (1999), con un método numérico.

\section{MODELO PROPUESTO PARA SECADO DE UN CILINDRO INFINITO}

Es desarrollado un modelo de secado, considerando una banana como un cilindro infinito, basándose en el análisis de parámetros, a partir del modelo de Luikov. En general el flujo de humedad, debido a gradientes de presión, no es significativo a temperaturas convencionales de secado. Por lo tanto, comúnmente los términos de presión pueden ser despreciados y las ecuaciones básicas para el análisis del secado son:

$$
\begin{aligned}
& \frac{\partial \mathrm{u}_{\mathrm{A}}}{\partial \mathrm{t}}=\nabla^{2}\left(\mathrm{~K}_{11} \mathrm{u}_{\mathrm{A}}\right)+\nabla^{2}\left(\mathrm{~K}_{12} \mathrm{~T}\right) \\
& \frac{\partial \mathrm{T}}{\partial \mathrm{t}}=\nabla^{2}\left(\mathrm{~K}_{21} \mathrm{u}_{\mathrm{A}}\right)+\nabla^{2}\left(\mathrm{~K}_{22} \mathrm{~T}\right)
\end{aligned}
$$

Siendo $\mathrm{u}_{\mathrm{A}}\left(\mathrm{kg}\right.$ de agua/kg de sólido) la humedad, $\mathrm{T}\left({ }^{\circ} \mathrm{C}\right)$ la temperatura, $\mathrm{t}(\mathrm{s})$ el tiempo, $\mathrm{K}_{\mathrm{ij}}, \mathrm{i}, \mathrm{j}, \mathrm{j}=1$ y 2 , son los coeficientes fenomenológicos para $\mathrm{i}=\mathrm{j}$ y los coeficientes combinados para $\mathrm{i} \neq \mathrm{j}$.

Los efectos de gradientes de temperatura en difusión de humedad sólo son significativos en el secado por conducción o cuando hay intenso calentamiento, tal como en secado por microondas o dieléctrico. Debido a eso, las ecuaciones fenomenológicas, se transforman en las siguientes, para un cilindro infinito, es decir, con transferencias solamente en la dimensión radial $r$ :

$$
\begin{aligned}
& \frac{\partial \mathrm{u}_{\mathrm{A}}}{\partial \mathrm{t}}=\frac{1}{\mathrm{r}} \frac{\partial}{\partial \mathrm{r}}\left(\mathrm{D}_{\mathrm{AB}} \mathrm{r} \frac{\partial \mathrm{u}_{\mathrm{A}}}{\partial \mathrm{r}}\right) \\
& \frac{\partial \mathrm{T}}{\partial \mathrm{t}}=\frac{1}{\mathrm{r}} \frac{\partial}{\partial \mathrm{r}}\left(\alpha \mathrm{r} \frac{\partial \mathrm{T}}{\partial \mathrm{r}}\right)
\end{aligned}
$$

Las Ecs.(6) y (7) son las ecuaciones difusivas de Fick y Fourier, respectivamente. Los parámetros $\mathrm{K}_{11}=$ $D_{A B}$ y $K_{22}=\alpha$ son, respectivamente, la difusividad del agua $D_{A B}$ y la difusividad térmica $\alpha=k /(\rho C p)$, y $\mathrm{Cp}\left(\mathrm{Jkg}^{-10} \mathrm{C}^{-1}\right)$ el calor específico de un fruto, $\mathrm{k}\left(\mathrm{Wm}^{-10} \mathrm{C}^{-1}\right)$ su conductividad térmica y $\rho\left(\mathrm{kg} / \mathrm{m}^{3}\right)$ su densidad.

Sea una fruta considerada como un cilindro infinito, de diámetro $D=2 R(m)$, con flujo unidimensional y simétrico de energía y materia en r. Dividiendo la Ec.(6) por la (7) y considerando constantes $K_{11}=D_{A B} y$ $\mathrm{K}_{22}=\alpha=\mathrm{k} /(\rho \mathrm{Cp})$, resulta de la adimensionalización de citadas ecuaciones, en: 


$$
\frac{\mathrm{D}_{\mathrm{AB}}}{\alpha}=\frac{1 / \alpha}{1 / \mathrm{D}_{\mathrm{AB}}}=\mathrm{Lu}=\frac{\frac{1}{\mathrm{r}} \frac{\partial}{\partial \mathrm{r}}\left(\mathrm{r} \frac{\partial \mathrm{T}}{\partial \mathrm{r}}\right) /(\partial \mathrm{T} / \partial \mathrm{t})}{\frac{1}{\mathrm{r}} \frac{\partial}{\partial \mathrm{r}}\left(\mathrm{r} \frac{\partial \mathrm{u}_{\mathrm{A}}}{\partial \mathrm{r}}\right) /\left(\partial \mathrm{u}_{\mathrm{A}} / \partial \mathrm{t}\right)}
$$

El número de Luikov es $L u=(1 / \alpha) /\left(1 / D_{A B}\right)=$ (resistencia interna a la difusión de calor $) /($ resistencia interna a la difusión de materia). Por ejemplo, a una temperatura de secado de $29,9{ }^{\circ} \mathrm{C}$, para una banana se calcula que $\mathrm{k}=0,481 \mathrm{Wm}^{-10} \mathrm{C}^{-1}, \rho=980 \mathrm{~kg} / \mathrm{m}^{3}$ y $\mathrm{Cp}=3.346 \mathrm{Jkg}^{-10} \mathrm{C}^{-1}$ y $\mathrm{D}_{\mathrm{AB}}=1,3510^{-10} \mathrm{~m}^{2} / \mathrm{s}$, resultando en $L u=D_{A B} / k /(\rho C p)=0,00092<<1$ (Costa y Ferreira, 2007; Costa, 2008). Cuando se cumple que Lu $<<$ 1 , significa que la transferencia interna de materia domina la transferencia simultánea de calor y materia, porque ella es una etapa mucho más lenta que la de transferencia de calor. Con las consideraciones realizadas y admitiendo, como primera aproximación, que no hay resistencia externa a la transferencia de energía, la Ec.(6) es la ecuación diferencial básica para el siguiente análisis.

Las condiciones de contorno para resolver la Ec.(6), que describe la transferencia de materia dentro de un cilindro infinito de radio $R(m)$, están dadas por:

$$
\begin{aligned}
& u_{A}(r ; 0)=u_{A 0} \quad t=0 \\
& -D_{A B} \rho_{S} \frac{d u_{A}}{d r}=0 \quad r=0 \\
& -D_{A B} \rho_{S} \frac{d u_{A}}{d r}=h_{m}\left(Y_{A S}-Y_{A \infty}\right) \quad r=R
\end{aligned}
$$

La Ec.(9) es la condición inicial, la Ec.(10) la de simetría y la Ec.(11) el flujo de materia en la superficie. En la Ec.(11) $Y_{A S}(\mathrm{~kg} / \mathrm{kg})$ es la concentración acuosa de equilibrio en la superficie de un fruto, $\mathrm{Y}_{\mathrm{A} \infty}$ la concentración acuosa en el medio externo lejos de la superficie, $h_{m}\left(\mathrm{kgm}^{-2} \mathrm{~s}^{-1}\right)=\mathrm{k}_{\mathrm{m}} \rho_{\mathrm{s}}$ un parámetro con unidad de flujo de materia, $\mathrm{k}_{\mathrm{m}}(\mathrm{m} / \mathrm{s})=\mathrm{h}_{\mathrm{m}} / \rho_{\mathrm{s}}$ el coeficiente externo de transferencia de materia y $\rho_{\mathrm{s}}\left(\mathrm{kg} / \mathrm{m}^{3}\right)$ la densidad de los sólidos en un fruto.

De la adimensionalización de la Ec.(11) se obtiene la ecuación siguiente, en la cual surge naturalmente el número de Biot de transferencia de materia $\mathrm{Bi}_{\mathrm{R}}$ :

$$
\mathrm{Bi}_{\mathrm{R}}=\frac{\mathrm{k}_{\mathrm{m}} \mathrm{R}}{\mathrm{D}_{\mathrm{AB}}}=\frac{\mathrm{h}_{\mathrm{m}} \mathrm{R}}{\mathrm{D}_{\mathrm{AB}} \rho_{\mathrm{S}}} \quad \mathrm{r}=\mathrm{R}
$$

El Biot, $B i_{R}=\left(1 / D_{A B} / R\right) /\left(1 / k_{m}\right)$, es la relación entre la resistencia interna a la transferencia de materia $1 / D_{A B} / R$ y la resistencia externa $1 / k_{m}$. Si Bi está en el rango $0,1<B i<100$, existe resistencia interna y externa a la transferencia de materia, cuando $\mathrm{Bi}<0,1$ no existe resistencia interna y si $\mathrm{Bi}>100$ no existe resistencia externa (Luikov, 1968).

La solución de la Ec.(6), con las condiciones de contorno de las Ec.(9) a (11), que da la humedad puntual de agua en una fruta $u_{A}(r ; t)$, o sea, la masa de agua/masa de sólidos en un fruto versus tiempo y posición (Crank, 1976), es:

$$
\begin{aligned}
& \frac{u_{A}-u_{A \infty}}{u_{A 0}-u_{A \infty}}=\sum_{n=1}^{\infty} A_{n} J_{0}\left(\mu_{n} r / R\right) \exp \left(-\mu_{n}^{2} D_{A B} t / R^{2}\right) \\
& B_{R} J 0\left(\mu_{n}\right)=\mu_{n} J_{1}\left(\mu_{n}\right)=\frac{k_{m} R}{D_{A B}} J_{0}\left(\mu_{n}\right)
\end{aligned}
$$




$$
A_{n}=\frac{2 B i_{R}}{J 0\left(\mu_{n}\right)\left(B i_{R}^{2}+\mu_{n}^{2}\right)}
$$

Siendo $u_{A \infty}$ la humedad a tiempo infinito. La Ec.(14) es la ecuación de autovalores $\mu_{n}$, que es auxiliar en la obtención de $u_{A}$ de la Ec.(13). En las ecuaciones anteriores, la función de Bessel de primera especie, de orden cero y uno, respectivamente, $\mathrm{J}_{0}\left(\mu_{n}\right)$ y $\mathrm{J}_{1}\left(\mu_{n}\right)$, son:

$$
\begin{aligned}
& J_{0}\left(\mu_{n}\right)=1-\frac{\mu_{n}^{2}}{2^{2}}+\frac{\mu_{n}^{4}}{2^{2} 4^{2}}-\frac{\mu_{n}^{6}}{2^{2} 4^{2} 6^{2}}+\ldots \\
& J_{1}\left(\mu_{n}\right)=\frac{\mu_{n}}{2}-\frac{\mu_{n}^{3}}{2^{2} 4}+\frac{\mu_{n}^{5}}{2^{2} 4^{2} 6}-\frac{\mu_{n}^{7}}{2^{2} 4^{2} 6^{2} 8}+\ldots
\end{aligned}
$$

Los $\mu_{\mathrm{n}}$ de la Ec.(14) son calculados usando las Ec.(16) y (17) como auxiliares; en función de $\mathrm{Bi}_{\mathrm{R}}=$ $k_{m} R / D_{A B}$. Los $\mu_{n}$ son encontrados en tablas (Crank, 1976; Luikov, 1968) o son calculados por programas como Excel o por correlaciones (Schwartzberg, 1981). Integrando la Ec.(13) de $r=0$ a $r=R$ en el volumen del cilindro, se obtiene la humedad total $U_{A}=M_{A} / M_{S}$; que es la relación entre la masa total de agua en una fruta $M_{A}$ y la masa total de sólidos $M_{S}$. Es decir, la integración es realizada sustituyendo la humedad $u_{A}$ de la Ec.(13) en la siguiente ecuación:

$$
U_{A}=\frac{\int u_{A} d V}{\int d V}=\frac{\int_{0}^{R} u_{A} r d r}{R^{2} / 2}
$$

Es mejor trabajar con la masa de agua $M_{A}$ que con $U_{A}$. Después de sustituir la Ec.(13) en la Ec.(18), además de $U_{A}=M_{A} / M_{S}, U_{A 0}=M_{A 0} / M_{S}$ y $U_{A \infty}=M_{A \infty} / M_{S}$, resulta de la integración:

$$
\begin{aligned}
& \frac{M_{A}-M_{A \infty}}{M_{A 0}-M_{A \infty}}=\sum_{n=1}^{\infty} B_{n} \exp \left(\frac{-\mu_{n}^{2} D_{A B} t}{R^{2}}\right) \\
& B_{n}=\frac{4 B_{R}{ }_{R}^{2}}{\mu_{n}^{2}\left(\mu_{n}^{2}+B_{R}^{2}\right)}
\end{aligned}
$$

Usando la Ec.(19) se calcula $M_{A}$ versus $t$, en función de Biot $B i_{R}$. Con la Ec.(19) también pueden ser evaluados $D_{A B}$ y $k_{m}$, si fueren conocidos $M_{A}$ versus $t$, y el radio $R$. La masa a tiempo infinito $M_{A \infty}$ coincide con la de equilibrio $\mathrm{M}_{\mathrm{Ae}}$, a una temperatura dada, presión y humedad ambiente. Si $\mathrm{M}_{\mathrm{A} \infty}$ es usada como un parámetro libre, al correlacionar $\mathrm{M}_{\mathrm{A}}$ versus $\mathrm{t}$, entonces ella puede no ser igual a $\mathrm{M}_{\mathrm{Ae}}$, debido al ajuste de parámetros.

\section{METODOLOGÍA DE CÁLCULO DE PARÁMETROS DEL MODELO PROPUESTO}

Es presentada una metodología para calcular parámetros, tales como $D_{A B}, B_{R}$ y $k_{m}$, basándose en la Ec.(19). Para realizar cálculos con la Ec.(19) o cuando se emplea solamente el primer autovalor $\mu_{1}$, de la Ec.(14), es conveniente usar una correlación en vez de resolver la serie de potencias de la Ec.(14). Con este objetivo, pueden ser empleadas las siguientes ecuaciones para $\mu_{1}$, en función de $\mathrm{Bi}$, presentadas por Schwartzberg (1981), para cilindro infinito: 


$$
\begin{aligned}
& \mu_{1}=\sqrt{\frac{2 \mathrm{Bi}}{(1+0,26 \mathrm{Bi})}} \text { Para } \mathrm{Bi}<2 \\
& \mu_{1}=\frac{2,4048 \mathrm{Bi}}{1,03+\mathrm{Bi}} \text { Para } \mathrm{Bi}>2
\end{aligned}
$$

El rango de Bi presentado por Schwartzberg (1981), para las Ec.(21) y (22) es, respectivamente, Bi $<3$ y $\mathrm{Bi}>5$. Las Ec.(21) y (22), respectivamente, en general dan desviaciones menores que $|1,5 \%|$, en el cálculo de $\mu_{1}$, en comparación con el evaluado con la solución exacta de la Ec.(14). Los cálculos con el modelo presentado se simplifican mucho, en parte, por usar una correlación como la Ec.(21) o (22); eliminando los, a veces, engorrosos procedimientos de cálculo con ecuaciones exactas de autovalores.

El modelo presentado es válido para todo el rango de Biot, $0<\mathrm{Bi}_{\mathrm{R}}<\infty$; pero si $\mathrm{Bi}_{\mathrm{R}}<0,1$ casi no hay interés en la práctica de secado de alimentos. Usando las ecuaciones de esta sección en el rango intermediario, $0,1<\mathrm{Bi}_{\mathrm{R}}<100$, que es el de mayor interés en secado, se calcula simultáneamente $\mathrm{Bi}_{\mathrm{R}} \mathrm{y}$ $D_{A B}$, y enseguida $k_{m}$.

El procedimiento de cálculo para evaluar los parámetros $B i_{R}, D_{A B}$ y $k_{m}$, cuando no hay disminución del radio de un cilindro infinito $R_{0}$, es:

i) Son obtenidos datos experimentales de $M_{A}$ versus $t$.

ii) Es reemplazada en el primer término de la Ec.(19), por ejemplo, la ecuación de $\mu_{1}$ de la Ec.(22), para $\mathrm{Bi}_{\mathrm{R}}>2$. Es decir, tanto en el factor preexponencial de la Ec.(19), dado por $\mathrm{B}_{1}$ de la Ec.(20), así como en el término dentro del exponencial, reemplazando el autovalor al cuadrado, esto es, elevando la Ec.(22) al cuadrado, $\mu_{1}{ }^{2}$. Después de esas sustituciones, resulta del primer término de la Ec.(19):

$$
\frac{M_{A}-M_{A \infty}}{M_{A 0}-M_{A \infty}}=\frac{4\left(1,03+B i_{R}\right)^{4}}{\left(2,4048 B i_{R}\right)^{2}\left\{2,4048^{2}+\left(1,03+B i_{R}\right)^{2}\right\}} \exp \left\{-\left(\frac{2,4048 B i_{R}}{1,03+B i_{R}}\right)^{2} \frac{D_{A B} t^{2}}{R^{2}}\right\}
$$

iii) Son correlacionados datos de $\mathrm{M}_{\mathrm{A}}$ versus t de la $\mathrm{Ec}$.(23), con un programa computacional de regresión no lineal, obteniendo directamente $B i_{R}$ y $D_{A B}$, y con estos valores y el radio $R_{0}$, se obtiene $k_{m}$ de la Ec.(12) $B i_{R}=k_{m} R_{0} / D_{A B}$. Es decir, son calculados simultáneamente $B i_{R}, D_{A B} y k_{m}$.

Si hay disminución de volumen, es necesario adicionar una ecuación relacionando la disminución del radio en el secado de un cilindro infinito $\mathrm{R}$, en función, por ejemplo, de la concentración acuosa (Queiroz y Nebra, 2001). Se propone correlacionar $R$ versus $M_{A}$, con la siguiente ecuación; siendo (a) y (b) parámetros obtenidos por regresión de datos:

$$
\mathrm{R}=\mathrm{a}+\mathrm{bM}_{\mathrm{A}}
$$

Nótese que en las Ecs.(23) y (24) existe la variable $\mathrm{M}_{\mathrm{A}}$. Después de insertar la Ec.(24) en la Ec.(23), se puede obtener simultáneamente $\mathrm{Bi}_{\mathrm{R}}$ y $\mathrm{D}_{\mathrm{AB}}$, admitiendo disminución del radio en el secado. Los valores de $M_{A 0}$ y $M_{A \infty}$ también pueden ser obtenidos como parámetros libres de la Ec.(23), si ellos no son conocidos.

Como en la Ec.(24) el radio $\mathrm{R}$ disminuye en el secado, puede ser usado un $\mathrm{R}_{\mathrm{m}}$ promedio para el proceso. Por ejemplo, la media aritmética del radio en el inicio y final del secado $t_{0}$ y $t_{f}$, respectivamente, resultando en $R_{m}=\left(R_{0}+R_{f}\right) / 2$, o un $R_{m}$ a tiempo promedio $t_{1 / 2}=\left(t_{0}+t_{f}\right) / 2$, obteniendo $R_{m}\left(t_{1 / 2}\right) \circ$ un $R_{m}$ integrando $R$ de la Ec.(24) de $t_{0} a t_{f}$ y dividiendo por $\left(t_{f}-t_{0}\right)$, resultando en $R_{m}=\int R d t /\left(t_{f}-t_{0}\right)$. El radio $R_{m}$ es un parámetro auxiliar en el procedimiento de cálculo a continuación, para evaluar $\mathrm{Bi}_{\mathrm{L}}$ y $\mathrm{k}_{\mathrm{m}}$ para un sólido esferoidal de longitud $2 \mathrm{~L}$, a partir de $\mathrm{Bi}_{\mathrm{R}}$ y $\mathrm{D}_{\mathrm{AB}}$ obtenidos para un cilindro infinito de diámetro $2 \mathrm{R}$. 
El procedimiento para calcular los parámetros $\mathrm{Bi}_{R}, \mathrm{D}_{\mathrm{AB}}$ y $\mathrm{k}_{\mathrm{m}}$, con disminución del radio de un cilindro infinito en el secado, es:

1) Son empleadas las siguientes variables en la $E c .(23): X=t / R^{2}$ versus $Y=\left(M_{A}-M_{A \infty}\right) /\left(M_{A 0}-M_{A \infty}\right)$; y el radio $\mathrm{R}$ es dado por la Ec.(24).

2) Son mantenidos como parámetros libres de un cilindro infinito $B_{R} y_{A B}$, que son calculados con un programa de correlación no lineal.

3) Es calculado un radio promedio $R_{m}$, por ejemplo, $R_{m}\left(t_{1 / 2}\right)$ a tiempo promedio $t_{1 / 2}=\left(t_{0}+t_{f}\right) / 2$.

4) Son usados el Bi para un cilindro infinito $B i_{R}=k_{m} R_{m} / D_{A B}$ de la Ec.(12), dividida por la Ec.(3) $B i_{L}=$ $k_{m} L / D_{A B}$, para calcular Bi para un sólido esferoidal $B i_{L}=B i_{R} L / R_{m}$; tanto en el inicio como en el final del proceso. Evaluando $L_{0}$ a $t_{0}=0$ y empleando $B_{R}$ y $R_{m}\left(t_{1 / 2}\right)$, se obtiene el Biot en el inicio, para un sólido esferoidal $\mathrm{Bi}_{\mathrm{LO}}=\mathrm{Bi}_{\mathrm{R}} \mathrm{L}_{0} / \mathrm{R}_{\mathrm{m}}$.

5) Son empleados $B i_{L 0}, L_{0}$ y $D_{A B}$, evaluando el coeficiente $k_{m 0}$ para un sólido esferoidal, en el inicio del secado, de la Ec. (3) $k_{m 0}=B i_{L 0} D_{A B} / L_{0}$.

6) Son usados $B i_{R}, R_{m}\left(t_{1 / 2}\right)$ y $L_{f}$ evaluado a $t=t_{f}$, calculando el Biot para un sólido esferoidal, en el final del proceso $B i_{L f}=B i_{R} L_{f} / R_{m}$.

7) Son empleados $B i_{L f}, L_{f}$ y $D_{A B}$, evaluando el coeficiente $k_{m f}$ para el sólido esferoidal, en el final del secado, de la Ec.(3) $\mathrm{k}_{\mathrm{mf}}=\mathrm{Bi}_{\mathrm{Lf}} \mathrm{D}_{\mathrm{AB}} / \mathrm{L}_{\mathrm{f}}$.

Algunos otros posibles procedimientos de cálculo son presentados a continuación. Como el Biot en el radio $B_{R}=k_{m} R / D_{A B}$, reemplazando $R$ de la $E c$.(24) en $B i_{R}$, sustituyendo el resultado en la Ec.(23) y correlacionando $M_{A}$ versus $t$, se obtiene $D_{A B}$ y $k_{m}$ como parámetros libres, en vez de $B i_{R}$ y $D_{A B}$. Si en la Ec.(23) están libres los términos $R, k_{m}$ y $D_{A B}$, se obtiene de esos parámetros un Biot $B i=k_{m} R_{m} / D_{A B}$ en función de un radio promedio $\mathrm{R}_{\mathrm{m}}$.

\section{RESULTADOS Y DISCUSIÓN}

Son realizados cálculos con el modelo analítico desarrollado y la metodología propuesta, usando datos de Queiroz (1994), ecuaciones y datos presentados por Lima (1999) y Lima et al. (2002); algunos de ellos reproducidos en la Tabla 1.

En la Tabla 1 son presentados datos de humedad de equilibrio $U_{e}$ (en base seca) $=M_{A e} / M_{S}$, siendo $M_{A e}$ la masa de agua en la fruta en estado de equilibrio, las dimensiones iniciales de una banana $L_{10}$ y $L_{20}$, y el tiempo de secado t, para experimentos de Queiroz (1994); excepto $L_{2}$, que fue calculada por Lima (1999). Los subíndices (0), (f) y (e) representan, respectivamente, datos en el inicio, final y en condición de equilibrio de secado $(t \rightarrow \infty)$; y UR la humedad relativa del aire de secado.

Es resuelto un ejemplo para el ensayo (4) de la Tabla 1, para ilustrar el empleo de la metodología de este artículo; en la cual se considera una banana como cilindro infinito y disminución de R durante su secado. Los resultados obtenidos son comparados con los de simulaciones de Lima (1999); en las cuales se considera que una fruta es un sólido esferoidal, con disminución de $L_{1}$ y $L_{2}$ en el proceso.

Para evaluar $M_{A}$ de la Ec.(23) es necesario conocer $M_{A 0}$ y $M_{A \infty} 0$ obtenerlas como parámetros libres, correlacionando datos de $\mathrm{M}_{\mathrm{A}}$ versus t. En este artículo se calcula $\mathrm{M}_{\mathrm{A}}$ y $\mathrm{M}_{\mathrm{A} \infty}$ a partir de datos de las Tablas 1 y 2, como es presentado a continuación. Son usadas ecuaciones de $M_{A}$ versus $t$ correlacionadas por Lima (1999), a partir de experimentos de Queiroz (1994); porque es más fácil emplear esas ecuaciones, que colectar puntos de gráficas de $\mathrm{M}_{\mathrm{A}}$ versus $\mathrm{t}$, como presentadas por Lima et al. (2002) y, después, correlacionarlos para obtener $\mathrm{M}_{\mathrm{A}}$ versus $\mathrm{t}$.

Lima et al. (2002) presentan curvas y datos experimentales de $M_{A}$ versus $t$, de los seis ensayos de la Tabla 1 y Lima (1999) presenta esas curvas y ecuaciones de $M_{A}$ versus $t$; como la Ec.(25), mostrada a seguir. Las ecuaciones presentadas por Lima (1999) están expresadas en humedad en base seca $\mathrm{U}_{\mathrm{A}}$ (b.s). Por ejemplo, de la Tabla 1, para el ensayo (4), la humedad inicial en base seca es $\mathrm{U}_{\mathrm{A} 0}=2,96=$ $\mathrm{M}_{\mathrm{A} 0} / \mathrm{M}_{\mathrm{S}}$ y en base húmeda $\mathrm{U}_{\mathrm{A} 0} /\left(\mathrm{U}_{\mathrm{A} 0}+1\right)=\mathrm{M}_{\mathrm{A} 0} /\left(\mathrm{M}_{\mathrm{A} 0}+\mathrm{M}_{\mathrm{S}}\right)=2,96 /(2,96+1)=0,7475$. Es decir, una banana contiene inicialmente $\mathrm{Mu}_{0}=74,75 \%$ de agua y $25,25 \%$ de sólidos. 
Se calcula la masa de una banana en inicio del secado, para el ensayo (4), partiendo de su volumen inicial $V_{0}$ y densidad $\rho_{0}$, resultando en $\rho_{0} V_{0}=M_{A 0}+M_{S}$. El volumen $V_{0}$ es calculado por la Ec.(2), usando las dimensiones iniciales del sólido esferoidal $L_{10} y L_{20}$, que están reproducidas en la Tabla 1, obteniendo $\mathrm{V}_{0}=57,8210^{-6} \mathrm{~m}^{3}$. Este volumen es similar al de la Tabla 2, $\mathrm{V}_{0}=57,830 \mathrm{~cm}^{3}$. Usando $\rho_{0}=980 \mathrm{~kg} / \mathrm{m}^{3}$, $\rho_{0} V_{0}=M_{A 0}+M_{S}=56,66 \mathrm{~g}$, resulta en $M_{A 0}=0,7475(56,66 \mathrm{~g})=42,36 \mathrm{~g} \mathrm{y} M_{S}=14,31 \mathrm{~g}$. Como $U_{A e}=$ $0,0426=U_{A \infty}=M_{A \infty} / 14,31 \mathrm{~g}$, se obtiene que $M_{A_{\infty}}=0,61 \mathrm{~g}$. Reemplazando esos valores en el lado izquierdo de la Ec.(23), resulta:

$$
\frac{M_{A}-0,61 \mathrm{~g}}{42,36 \mathrm{~g}-0,61 \mathrm{~g}}=1,5988845 \exp (-0,077787 \mathrm{t})-0,2887315 \exp (-0,0935546 \mathrm{t})-0,3137324 \exp (-0,0680589 \mathrm{t})
$$

El lado derecho de la Ec.(25) fue obtenido por Lima (1999), de datos $M_{A}$ versus t de Queiroz (1994); y presenta muchas cifras decimales en cada término. El lado izquierdo de la Ec.(25) fue calculado en este artículo porque él es necesario en cálculos posteriores, por ejemplo, para evaluar $M_{A}$ y $R$.

Si no hubiera disminución de volumen, el lado derecho de la Ec.(25) podría ser igualado al derecho de la Ec.(23), usando el radio inicial $R=R_{0}$, generando puntos a cada t, obteniendo $B i_{R}$ y $D_{A B} y$, enseguida, $B i_{L}$ $=\mathrm{Bi}_{R} \mathrm{~L} / \mathrm{R}$ y $\mathrm{k}_{\mathrm{m}}=\mathrm{Bi}_{L} \mathrm{D}_{\mathrm{AB}} / \mathrm{L}$. Pero como hay disminución de $\mathrm{R}$, son usadas las Ec.(23) y (24), con el procedimiento ya descrito y que es implementado a seguir.

En la Tabla 2 son presentados datos de disminución de dimensiones de una banana, para los ensayos de la Tabla 1. Los datos de la Tabla 2 para el inicio y el final del secado, son área superficial de una banana $S$, su volumen $V$, sus dimensiones $L_{1}$ y $L_{2}$, y el coeficiente adimensional de encogimiento o de disminución de volumen $\bar{\beta}$. Éste coeficiente está asociado con la modificación de volumen sufrida por un sólido durante la difusión de agua. Si $\bar{\beta}=0,70$ significa que su volumen al final del proceso, cuando se alcanza su humedad de equilibrio, será $30 \%$ de su volumen inicial. Para el ensayo (4) el volumen inicial $\mathrm{y}$ final son, respectivamente, $\mathrm{V}_{0}=57,830 \mathrm{~cm}^{3}$ y $\mathrm{V}_{\mathrm{f}}=14,869 \mathrm{~cm}^{3}$, resultando en $\beta=1-\mathrm{V}_{\mathrm{f}} / \mathrm{V}_{0}=0,7429$, que es similar a $\bar{\beta}=0,7844$; el promedio de la Tabla 2.

Tabla 1: Parámetros para aire y banana de los ensayos de secado (Lima, 1999; Queiroz, 1994).

\begin{tabular}{|c|c|c|c|c|c|c|c|c|c|c|c|}
\hline \multirow{2}{*}{ Ensayo } & \multicolumn{3}{|c|}{ Datos del aire } & \multicolumn{9}{|c|}{ Datos de una banana } & Tiempo de \\
\cline { 2 - 12 } & $\begin{array}{c}\mathrm{T}_{a} \\
\left({ }^{\circ} \mathrm{C}\right)\end{array}$ & $\begin{array}{c}\mathrm{UR} \\
(\%)\end{array}$ & $\begin{array}{c}\mathrm{v} \\
(\mathrm{m} / \mathrm{s})\end{array}$ & $\begin{array}{c}\mathrm{U}_{0} \\
(\mathrm{~b} . \mathrm{s})\end{array}$ & $\begin{array}{c}\mathrm{U}_{\mathrm{f}} \\
(\mathrm{b} . \mathrm{s})\end{array}$ & $\begin{array}{c}\mathrm{U}_{\mathrm{e}} \\
(\mathrm{b} . \mathrm{s})\end{array}$ & $\begin{array}{c}\mathrm{T}_{0} \\
\left({ }^{\circ} \mathrm{C}\right)\end{array}$ & $\begin{array}{c}\mathrm{T}_{\mathrm{f}} \\
\left({ }^{\circ} \mathrm{C}\right)\end{array}$ & $\begin{array}{c}\mathrm{L}_{20} \\
(\mathrm{~m})\end{array}$ & $\begin{array}{c}\mathrm{L}_{10} \\
(\mathrm{~m})\end{array}$ & $\begin{array}{c}\text { secado } \\
(\mathrm{h})\end{array}$ \\
\hline 1 & 29,9 & 35,7 & 0,38 & 3,43 & 0,32 & 0,1428 & 19,1 & 29,7 & 0,05856 & 0,01613 & 121,85 \\
\hline 2 & 39,9 & 19,3 & 0,33 & 3,17 & 0,33 & 0,0664 & 21,0 & 38,9 & 0,05878 & 0,01569 & 72,00 \\
\hline 3 & 49,9 & 19,2 & 0,37 & 3,21 & 0,32 & 0,0579 & 20,3 & 47,1 & 0,05901 & 0,01522 & 40,80 \\
\hline 4 & 60,2 & 19,9 & 0,36 & 2,96 & 0,25 & 0,0426 & 30,6 & 57,5 & 0,05897 & 0,01530 & 35,3 \\
\hline 5 & 60,5 & 10,7 & 0,35 & 3,04 & 0,31 & 0,0211 & 23,4 & 57,0 & 0,05909 & 0,01506 & 27,80 \\
\hline 6 & 68,4 & 7,3 & 0,39 & 2,95 & 0,22 & 0,0121 & 25,3 & 64,2 & 0,05890 & 0,01545 & 27,60 \\
\hline
\end{tabular}

Tabla 2: Dimensiones de una banana en su secado y coeficiente de disminución de volumen (Lima, 1999; Queiroz, 1994).

\begin{tabular}{|c|c|c|c|c|c|c|c|c|c|c|c|c|}
\hline$E$ & \multicolumn{3}{|c|}{ Datos del aire } & \multicolumn{4}{|c|}{ Inicio del secado } & \multicolumn{4}{|c|}{ Final del secado } & \multirow[b]{2}{*}{$\beta$} \\
\hline a & $\begin{array}{c}\mathrm{T}_{\mathrm{a}} \\
\left({ }^{\circ} \mathrm{C}\right)\end{array}$ & $\begin{array}{l}\text { UR } \\
(\%)\end{array}$ & $\begin{array}{c}\mathrm{V} \\
(\mathrm{m} / \mathrm{s})\end{array}$ & $\begin{array}{l}\mathrm{L}_{10} \\
(\mathrm{~cm})\end{array}$ & $\begin{array}{l}\mathrm{L}_{20} \\
(\mathrm{~cm})\end{array}$ & $\begin{array}{c}\mathrm{V}_{0} \\
\left(\mathrm{~cm}^{3}\right)\end{array}$ & $\begin{array}{c}\mathrm{S}_{0} \\
\left(\mathrm{~cm}^{2}\right)\end{array}$ & $\begin{array}{c}\mathrm{L}_{1 f} \\
(\mathrm{~cm})\end{array}$ & $\begin{array}{l}\mathrm{L}_{2 f} \\
(\mathrm{~cm})\end{array}$ & $\begin{array}{c}V_{f} \\
\left(\mathrm{~cm}^{3}\right)\end{array}$ & $\begin{array}{c}\mathrm{S}_{\mathrm{f}} \\
\left(\mathrm{cm}^{2}\right)\end{array}$ & \\
\hline 1 & 29,9 & 35,7 & 0,38 & 1,6130 & 5,8562 & 63,822 & 96,099 & 0,8614 & 3,1274 & 9,720 & 27,407 & 0,8838 \\
\hline 2 & 39,9 & 19,3 & 0,33 & 1,5690 & 5,8784 & 60,616 & 93,676 & 0,9457 & 3,5433 & 13,275 & 34,035 & 0,8345 \\
\hline 3 & 49,9 & 19,2 & 0,37 & 1,5220 & 5,9016 & 57,265 & 91,074 & 0,9001 & 3,4903 & 11,846 & 31,856 & 0,8475 \\
\hline 4 & 60,2 & 19,9 & 0,36 & 1,530 & 5,8977 & 57,830 & 91,518 & 0,9729 & 3,7503 & 14,869 & 37,006 & 0,7844 \\
\hline 5 & 60,5 & 10,7 & 0,35 & 1,5060 & 5,9095 & 56,142 & 90,184 & 0,9481 & 3,7205 & 14,010 & 35,746 & 0,8117 \\
\hline 6 & 68,4 & 7,3 & 0,39 & 1,5450 & 5,8903 & 58,896 & 92,349 & 0,9915 & 3,7800 & 15,565 & 38,032 & 0,7899 \\
\hline
\end{tabular}


Se correlaciona $R$ versus $M_{A}$ de la Ec.(24), usando datos para el ensayo (4) de la Tabla 2. Se obtiene de la Tabla 2, respectivamente, para el inicio y final del proceso, como primera aproximación que $R_{0} \cong L_{10}=$ $0,01530 \mathrm{~m}$ y $R_{f} \cong L_{1 f}=0,009729 \mathrm{~m}$. Pero como $M_{A 0}=42,36 \mathrm{~g}, M_{S}=14,31 \mathrm{~g}$, y en el final del secado, de la Tabla 1, $U_{f}=0,25=M_{A f} / M_{S}$, resulta en $M_{A f}=3,58 \mathrm{~g}$. Con esos valores sustituidos en la Ec.(24), la disminución de $R \cong L_{1}$ versus $M_{A}$, para $R_{0}=0,01530 \mathrm{~m}$, está dado por:

$$
R=R_{0}-\frac{\left(R_{0}-R_{f}\right)}{\left(M_{A 0}-M_{A f}\right)} M_{A 0}+\frac{\left(R_{0}-R_{f}\right)}{\left(M_{A 0}-M_{A f}\right)} M_{A}=R_{0}-\frac{\left(R_{0}-R_{f}\right)}{\left(M_{A 0}-M_{A f}\right)}\left(M_{A 0}-M_{A}\right)=0,01530-0,0001436\left(42,36-M_{A}\right)
$$

De las Ecs.(24) y (26), resulta en $a=\{0,01530-0,0001436(42,36)\} \mathrm{m}$ y b $=0,0001436 \mathrm{~m} / \mathrm{g}$. Para $\mathrm{t}_{0}=0 \mathrm{~h}$ y $\mathrm{M}_{\mathrm{A} 0}=42,36 \mathrm{~g}$, de la Ec.(26) resulta $\mathrm{R}=\mathrm{R}_{0}$ y a $\mathrm{t}_{\mathrm{f}}=35,3 \mathrm{~h}$, de la Ec.(25) $\mathrm{M}_{\mathrm{A}}=3,27 \mathrm{~g}$ y de la Ec. (26) $R_{\mathrm{f}}=$ $L_{1 f}=0,009686 \mathrm{~m}$; que da una desviación de $-0,44 \%$ en relación a $R_{f} \cong L_{1 f}=0,009729 \mathrm{~m}$, de la Tabla 2 . Una ecuación similar a la Ec.(26) puede ser obtenida para disminución de $L_{2}$, si una banana es considerada un cilindro finito. Nótese en la Ec.(26) que R disminuye debido a la disminución de $\mathrm{M}_{\mathrm{A}}$ en la fruta. Las $\mathrm{M}_{\mathrm{A}}$ de la Ec.(26) pueden ser experimentales o de una ecuación como, por ejemplo, la Ec. (25). Es decir, de $\mathrm{M}_{\mathrm{A}}$ experimentales o generadas por la Ec.(25) se estudia la disminución en $\mathrm{R}$, de la Ec.(26).

El procedimiento para obtener los parámetros $\mathrm{Bi}_{\mathrm{R}}, \mathrm{D}_{\mathrm{AB}}$ y $\mathrm{k}_{\mathrm{m}}$, para el ensayo (4), con disminución del radio de una fruta, es:

i) Obtener $Y=\left(M_{A}-M_{A \infty}\right) /\left(M_{A 0}-M_{A \infty}\right)$ usando valores de $t_{0}=0 h$ a $t_{f}=35,3 h$, en el lado derecho de la Ec.(25); resultando en la columna (2) de la Tabla 3.

ii) Reordenar la Ec.(25) para obtener $\mathrm{M}_{\mathrm{A}}$ versus t; obteniendo la columna (3) de la Tabla 3. El término $\mathrm{Y}$ es el valor calculado en la columna (2).

iii) Sustituir las $M_{A}$ de la columna (3), en $R$ de la Ec.(26); resultando en la columna (4).

iv) Obtener $X=t / R^{2}$, dividiendo la columna (1), $t$, por la columna (4) al cuadrado, $R^{2}$; resultando en la columna (5).

v) Correlacionar $X=t / R^{2}$ versus $Y=\left(M_{A}-M_{A \infty}\right) /\left(M_{A 0}-M_{A \infty}\right)$ de la Ec.(23), es decir, las columnas (5) y (2) de la Tabla 3, con el programa de regresión no lineal Lab Fit (Silva y Silva, 2003), usando como parámetros libres $\mathrm{Bi}_{R}$ y $D_{A B}$, resultando en:

$$
\frac{M_{A}-0,61 \mathrm{~g}}{42,36 \mathrm{~g}-0,61 \mathrm{~g}}=0,910 \exp \left\{-(1,833)^{2}\left(0,2501.10^{-5}\right) \frac{\mathrm{t}}{\mathrm{R}^{2}}\right\}
$$

vi) Del Lab Fit para la Ec.(27), presentada en la Fig.(2), se obtuvo $D_{A B}=0,250110^{-5} \mathrm{~m}^{2} / \mathrm{h}=6,9510^{-10} \mathrm{~m}^{2} / \mathrm{s}$, $R^{2} y y(x)=0,987$, Biot en el radio $B i_{R}=3,299 \cong 3,30$ y $\mu_{1}=1,833$. Los parámetros $D_{A B}$ y $B_{R}$ son presentados en las Tablas 4 y 5 . El $\mu_{1}$ es calculado de la Ec.(22) o por el término correspondiente dentro del exponencial de la Ec.(27). La $D_{A B}=6,9510^{-10} \mathrm{~m}^{2} / \mathrm{s}$ difiere en $-4,2 \%$ de la $D_{A B}=7,2510^{-10}$ $\mathrm{m}^{2} / \mathrm{s}$ de Lima (1999), para el ensayo (4) de la Tabla 5 y modelo (III), con convección y disminución de $L_{1}$ y $L_{2}$ de una banana, en aire a $60,2^{\circ} \mathrm{C}$ y $19,9 \%$ de humedad. En la Fig.(2) no aparecen todas las cifras decimales de la Ec.(27), porque en el Lab Fit sólo es posible escribir ecuaciones con hasta 94 cifras; por ejemplo, 2,4048 está escrito como 2,4.

vii)Usar el tiempo promedio $t_{1 / 2}=\left(t_{0}+t_{f}\right) / 2=35,3 \mathrm{~h} / 2=17,65 \mathrm{~h}$, para calcular $\mathrm{M}_{\mathrm{A}}\left(\mathrm{t}_{1 / 2}\right)=11,27 \mathrm{~g}$ y $\mathrm{R}_{\mathrm{m}}\left(\mathrm{t}_{1 / 2}\right)$ $=0,01084 \mathrm{~m}$, de las Ecs.(25) y (26), respectivamente. El radio promedio $R_{m}$ es empleado como una referencia en los cálculos a continuación, para obtener $\mathrm{Bi}_{\mathrm{L}}$ y $\mathrm{k}_{\mathrm{m}}$, en el inicio y final del proceso. Los valores de $t_{1 / 2}, M_{A}\left(t_{1 / 2}\right)$ y $R_{m}\left(t_{1 / 2}\right)$ están presentados en la Tabla 4.

viii)Dividir $\mathrm{Bi}_{\mathrm{R}}$ de la $\mathrm{Ec}$.(12), para cilindro infinito, por la $\mathrm{Ec}$.(3), para evaluar $\mathrm{Bi}_{\llcorner}$de un sólido esferoidal, si es conocido $L ;$ usando $B i_{L}=B i_{R} L / R_{m}$. Para el inicio del secado se obtiene para el sólido esferoidal, $L_{0}$ 
de la Ec.(1), usando $R_{0} \cong L_{10}$ y $L_{20}$ del ensayo (4) de la Tabla 2, así como Bi $i_{L 0}$, respectivamente, por las siguientes ecuaciones:

$$
\begin{aligned}
& \mathrm{L}_{0}=\sqrt{\left(\mathrm{L}_{20}^{2}-\mathrm{L}_{10}^{2}\right)}=\sqrt{(0,058977 \mathrm{~m})^{2}-(0,01530 \mathrm{~m})^{2}}=0,05696 \mathrm{~m} \\
& \mathrm{Bi}_{\mathrm{L} 0}=\mathrm{Bi}_{\mathrm{R}} \frac{\mathrm{L}_{0}}{\mathrm{R}_{\mathrm{m}}} \cong 3,30\left(\frac{0,05696}{0,01084}\right) \cong 17,35
\end{aligned}
$$

ix) El Bi de la Ec.(29) o (3) $\mathrm{Bi}_{\llcorner 0}=17,35$ presentado en la Tabla 4, difiere en $-1 \%$ de $B \mathrm{i}_{\llcorner 0}=17,52$ del ensayo (4) de la Tabla 5 y modelo (III). Lo mismo puede ser realizado para el final del proceso a $t_{f}=$ 35,3 h. De la Tabla 2 se obtiene $R_{f} \cong L_{1 f}$ y $L_{2 f}$, y se calcula $L_{f}$ y $B i_{L f}$, respectivamente, de las ecuaciones a continuación:

$$
\begin{aligned}
& L_{f}=\sqrt{\left(L_{2 f}^{2}-L_{1 f}^{2}\right)}=\sqrt{(0,037503 m)^{2}-(0,009729 m)^{2}}=0,03622 m \\
& B_{L f}=B i_{R} \frac{L_{f}}{R_{m}} \cong 3,30\left(\frac{0,03622}{0,01084}\right) \cong 11,03
\end{aligned}
$$

x) Evaluar $k_{m}$, de la Ec.(3), para el inicio del secado, empleando $D_{A B}, L_{0}$ y $B i_{\llcorner 0}$, de las Ec.(27), (28) y (29), respectivamente, resultando en $\mathrm{k}_{\mathrm{m} 0}$ :

$$
\mathrm{k}_{\mathrm{m} 0}=\frac{\mathrm{Bi}_{\mathrm{LO}} \mathrm{D}_{\mathrm{AB}}}{\mathrm{L}_{0}} \cong \frac{17,34\left(6,9510^{-10} \mathrm{~m}^{2} / \mathrm{s}\right)}{0,05696 \mathrm{~m}} \cong 21,16 \cdot 10^{-8} \mathrm{~m} / \mathrm{s}
$$

xi) Calcular $k_{m f}$ para el final del proceso, con $D_{A B}, L_{f}$ y $B i_{L f}$, de las Ec.(27), (30) y (31), respectivamente, resultando en:

$$
k_{m f}=\frac{B i_{L f} D_{A B}}{L_{f}} \cong \frac{11,03\left(6,9510^{-10} \mathrm{~m}^{2} / \mathrm{s}\right)}{0,03622 \mathrm{~m}} \cong 21,16 \cdot 10^{-8} \mathrm{~m} / \mathrm{s}
$$

En la Tabla 4 se presenta un resumen de los parámetros evaluados para el ensayo (4); acorde a la secuencia de cálculo (i) a (xi). El cálculo a continuación es realizado sólo para confirmar el valor de $\mathrm{k}_{\mathrm{m}}=$ $22,3010^{-8} \mathrm{~m} / \mathrm{s}$, presentado para el modelo (III) de la Tabla 5 . El $\mathrm{k}_{\mathrm{m}}$ para el ensayo (4), en el inicio del proceso, con $\mathrm{Bi}_{\mathrm{L} 0}=17,52, \mathrm{~L}_{0}=0,05696 \mathrm{~m}$ y $\mathrm{D}_{\mathrm{AB}}=7,2510^{-10} \mathrm{~m}^{2} / \mathrm{s}$, es $\mathrm{k}_{\mathrm{m} 0}=\mathrm{Bi}_{\mathrm{LO}} \mathrm{D}_{\mathrm{AB}} / \mathrm{L}=22,3010^{-8} \mathrm{~m} / \mathrm{s}$. Para el final del secado, con $B_{L f}=11,14, L_{f}=0,03622 \mathrm{~m} \mathrm{y}_{A B}=7,2510^{-10} \mathrm{~m}^{2} / \mathrm{s}$, resulta en $\mathrm{k}_{\mathrm{mf}}=22,3010^{-}$ ${ }^{8} \mathrm{~m} / \mathrm{s}$. El $\mathrm{k}_{\mathrm{m} 0}=21,1610^{-8} \mathrm{~m} / \mathrm{s}$ de este trabajo difiere en $-5,1 \%$ de $\mathrm{k}_{\mathrm{m} 0}=22,3010^{-8} \mathrm{~m} / \mathrm{s}$ del modelo (III).

Si no fuera considerada la disminución del radio $R$, esto es, usando el radio inicial $R_{0}=0,01530 \mathrm{~m}$ en la Ec.(23), correlacionando $X=t$ versus $Y=\left(M_{A}-M_{A \infty}\right) /\left(M_{A 0}-M_{A_{\infty}}\right)$, de las columnas (1) y (2) de la Tabla 3 , para el ensayo (4), resultaría en $D_{A B}=0,856810^{-5} \mathrm{~m}^{2} / \mathrm{h}=23,8010^{-10} \mathrm{~m}^{2} / \mathrm{s}$ y $B i_{R}=1,53$. Con $L_{0}=0,05696$ $\mathrm{m}$ de la Ec.(28) y $R_{0}=0,01530 \mathrm{~m}$, se obtiene de la Ec.(29) que $B_{\mathrm{L}}=5,70$ y de la Ec.(32) resulta en $\mathrm{k}_{\mathrm{m}}=$ $23,8010^{-8} \mathrm{~m} / \mathrm{s}$. La $D_{A B}=23,8010^{-10} \mathrm{~m}^{2} / \mathrm{s}$ es $-8 \%$ menor que $D_{A B}=25,8710^{-10} \mathrm{~m}^{2} / \mathrm{s}$, para condición de contorno convectiva, pero sin disminución de volumen, que es denominado modelo (II); que es presentado en la Tabla 5. Las $D_{A B}=23,8010^{-10} \mathrm{~m}^{2} / \mathrm{s}$ y $D_{A B}=25,8710^{-10} \mathrm{~m}^{2} / \mathrm{s}$ son muy diferentes de $D_{A B}=$ $6,9510^{-10} \mathrm{~m}^{2} / \mathrm{s}$, con disminución en R. El $\mathrm{k}_{\mathrm{m}}=23,8010^{-8} \mathrm{~m} / \mathrm{s}$ evaluado en esta publicación, sin considerar disminución en $\mathrm{R}$, es $12,6 \%$ mayor que $\mathrm{k}_{\mathrm{m} 0}=21,1310^{-8} \mathrm{~m} / \mathrm{s}$ con disminución en $\mathrm{R}$ y $39,8 \%$ mayor que $\mathrm{k}_{\mathrm{m}}=17,0310^{-8} \mathrm{~m} / \mathrm{s}$ del modelo (II).

De los resultados anteriores se concluye que para el ensayo (4), la $D_{A B}$ calculada en esta investigación concuerda bien con la evaluada por los modelos (II) y (III). También es buena la concordancia entre el $\mathrm{k}_{\mathrm{m}}$ calculado en este trabajo y por el modelo (III), pero no lo es por el modelo (II). 
Tabla 3: Parámetros de cálculo para el ensayo (4), cuando hay disminución del radio de una banana.

\begin{tabular}{|c|c|c|c|c|}
\hline$t(h)$ & $\begin{aligned} & Y= \frac{M_{A}-M_{A \infty}}{M_{A 0}-M_{A \infty}} \\
& E c .(25)\end{aligned}$ & $\begin{array}{c}M_{A}=M_{A \infty}+\left(M_{A 0}-M_{A \infty}\right) \cdot\{Y\} \\
M_{A}(g)=0,61 g+(42,36 g-0,61 g) \cdot\{Y\} \\
\text { Ec. }(25)\end{array}$ & $\begin{array}{l}\mathrm{R}(\mathrm{m}) \\
\text { Ec. }(26)\end{array}$ & $\begin{array}{c}\qquad X=\frac{t}{R^{2}} \\
\text { Columnas (1) y (4) }\end{array}$ \\
\hline 0,00 & 0,996 & 42,21 & 0,01528 & 0,000 \\
\hline 1,00 & 0,923 & 39,15 & 0,01484 & 4541,098 \\
\hline 2,00 & 0,855 & 36,32 & 0,01443 & 9602,049 \\
\hline 3,00 & 0,792 & 33,69 & 0,01405 & 15187,834 \\
\hline 4,00 & 0,734 & 31,25 & 0,01370 & 21299,284 \\
\hline 5,00 & 0,680 & 28,98 & 0,01338 & 27933,152 \\
\hline 6,00 & 0,629 & 26,88 & 0,01308 & 35082,263 \\
\hline 7,00 & 0,583 & 24,94 & 0,01280 & 42735,755 \\
\hline 8,00 & 0,540 & 23,14 & 0,01254 & 50879,372 \\
\hline 9,00 & 0,499 & 21,46 & 0,01230 & 59495,815 \\
\hline 10,00 & 0,462 & 19,91 & 0,01208 & 68565,132 \\
\hline 11,00 & 0,428 & 18,48 & 0,01187 & 78065,134 \\
\hline 12,00 & 0,396 & 17,15 & 0,01168 & 87971,820 \\
\hline 13,00 & 0,367 & 15,91 & 0,01150 & 98259,803 \\
\hline 14,00 & 0,339 & 14,77 & 0,01134 & 108902,729 \\
\hline 15,00 & 0,314 & 13,71 & 0,01119 & 119873,668 \\
\hline 16,00 & 0,290 & 12,73 & 0,01105 & 131145,481 \\
\hline 17,00 & 0,269 & 11,82 & 0,01092 & 142691,159 \\
\hline 18,00 & 0,248 & 10,98 & 0,01079 & 154484,110 \\
\hline 19,00 & 0,230 & 10,20 & 0,01068 & 166498,427 \\
\hline 20,00 & 0,213 & 9,48 & 0,01058 & 178709,094 \\
\hline 21,00 & 0,197 & 8,82 & 0,01048 & 191092,173 \\
\hline 22,00 & 0,182 & 8,20 & 0,01039 & 203624,933 \\
\hline 23,00 & 0,168 & 7,63 & 0,01031 & 216285,964 \\
\hline 24,00 & 0,155 & 7,10 & 0,01024 & 229055,240 \\
\hline 25,00 & 0,144 & 6,61 & 0,01017 & 241914,168 \\
\hline 26,00 & 0,133 & 6,15 & 0,01010 & 254845,597 \\
\hline 27,00 & 0,123 & 5,73 & 0,01004 & 267833,816 \\
\hline 28,00 & 0,113 & 5,34 & 0,00998 & 280864,530 \\
\hline 29,00 & 0,105 & 4,99 & 0,00993 & 293924,819 \\
\hline 30,00 & 0,097 & 4,65 & 0,00989 & 307003,083 \\
\hline 31,00 & 0,089 & 4,35 & 0,00984 & 320088,985 \\
\hline 32,00 & 0,083 & 4,06 & 0,00980 & 333173,380 \\
\hline 33,00 & 0,076 & 3,80 & 0,00976 & 346248,240 \\
\hline 34,00 & 0,071 & 3,56 & 0,00973 & 359306,581 \\
\hline 35,00 & 0,065 & 3,33 & 0,00970 & 372342,387 \\
\hline 35,30 & 0,064 & 3,27 & 0,00969 & 376247,945 \\
\hline
\end{tabular}

Tabla 4: Parámetros calculados con el modelo analítico, para los ensayos (1) al (6).

\begin{tabular}{|c|c|c|c|c|c|c|c|c|c|c|c|}
\hline a & $\begin{array}{c}D_{A B} \cdot 10^{10} \\
\left(\mathrm{~m}^{2} / \mathrm{s}\right)\end{array}$ & $\begin{array}{c}\mathrm{Bi}_{\mathrm{R}} \\
\text { (adim.) }\end{array}$ & $\begin{array}{l}\mathrm{L}_{0} \\
(\mathrm{~m})\end{array}$ & $\begin{array}{l}\mathrm{L}_{\mathrm{f}} \\
(\mathrm{m})\end{array}$ & $\begin{array}{l}t_{1 / 2} \\
(h)\end{array}$ & $\begin{array}{c}M_{A}\left(t_{1 / 2}\right) \\
(g)\end{array}$ & $\begin{array}{c}\mathrm{R}_{\mathrm{m}}\left(\mathrm{t}_{1 / 2}\right) \\
(\mathrm{m})\end{array}$ & $\begin{array}{c}\mathrm{Bi}_{\llcorner 0} \\
\text { (adim.) }\end{array}$ & $\begin{array}{c}\mathrm{Bi}_{\mathrm{Lf}} \\
\text { (adim.) }\end{array}$ & $\begin{array}{c}\mathrm{k}_{\mathrm{m} 0} \cdot 10^{8} \\
(\mathrm{~m} / \mathrm{s})\end{array}$ & $\begin{array}{r}k_{m f} \cdot 10^{8} \\
(\mathrm{~m} / \mathrm{s})\end{array}$ \\
\hline 1 & 1,35 & 6,62 & 0,05630 & 0,03006 & 60,93 & 13,60 & 0,01015 & 36,72 & 19,61 & 8,80 & 8,80 \\
\hline 2 & 2,32 & 5,97 & 0,05665 & 0,03415 & 36,00 & 13,29 & 0,01078 & 31,38 & 18,91 & 12,86 & 12,86 \\
\hline 3 & 4,01 & 4,96 & 0,05702 & 0,03372 & 20,40 & 12,69 & 0,01036 & 27,29 & 16,14 & 19,17 & 19,17 \\
\hline 4 & 6,95 & 3,30 & 0,05696 & 0,03622 & 17,65 & 11,27 & 0,01084 & 17,35 & 11,03 & 21,16 & 21,16 \\
\hline 5 & 6,71 & 3,91 & 0,05714 & 0,03598 & 13,90 & 12,49 & 0,01072 & 20,84 & 13,12 & 24,46 & 24,46 \\
\hline 6 & 9,69 & 2,67 & 0,05684 & 0,03648 & 13,80 & 12,36 & 0,01118 & 13,57 & 8,71 & 23,15 & 23,15 \\
\hline
\end{tabular}

En las Tablas 4 y 5 se presentan los principales resultados de este trabajo, para los ensayos (1) al (6), de igual manera a la desarrollada para el ensayo (4), con ecuaciones similares as las Ecs.(25) y (26), 
resultando en la Ec.(27); que es empleada para evaluación de parámetros. Fueron usadas ecuaciones similares al lado derecho de la Ec.(25) de $M_{A}$ versus $t$, para los otros ensayos, presentadas por Lima (1999) y reproducidas en la Tabla 6. Para el lado izquierdo de ecuaciones del tipo de la Ec.(25), fueron calculadas $M_{A 0}$ y $M_{A \infty}$, con datos de la Tabla 1, $\rho_{0}=980 \mathrm{~kg} / \mathrm{m}^{3}$ y el procedimiento descrito antes de dicha ecuación. Fueron obtenidas ecuaciones similares a la Ec.(26) de $R$ versus $M_{A}$, con el procedimiento descrito antes de mencionada ecuación. Un resumen de las ecuaciones básicas de cálculo para todos los ensayos, es presentado en la Tabla 6. Ellas son usadas para obtener ecuaciones como la Ec.(27) y calcular los parámetros de las Tablas 4 y 5 .

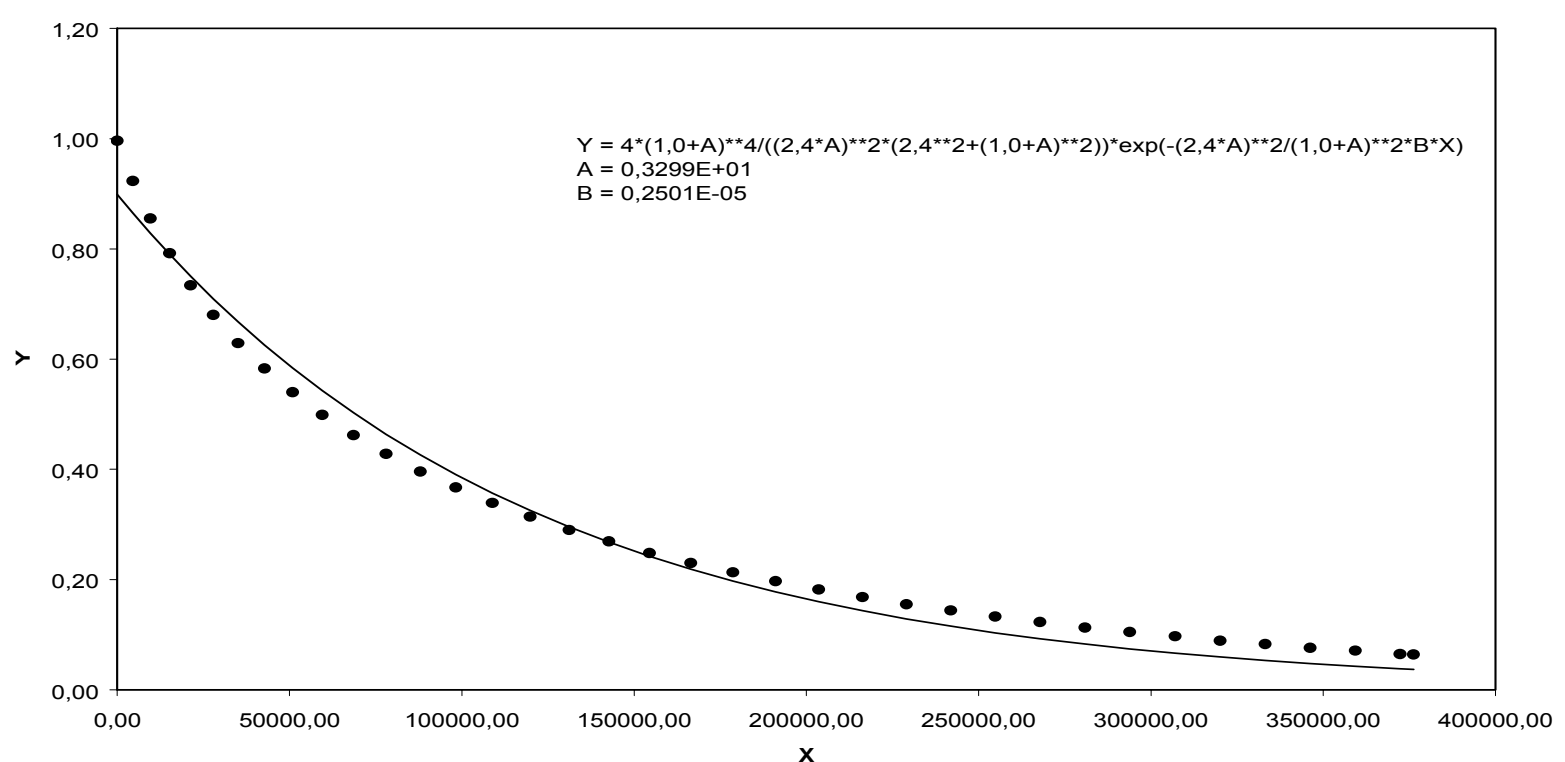

Fig.2. Masa adimensional de agua en banana $Y=\left(M_{A}-M_{A \infty}\right) /\left(M_{A 0}-M_{A \infty}\right)=\left(M_{A}-M_{A i n f}\right) /\left(M_{A 0}-M_{A i n f}\right)$ versus $X=t / R^{2}$, para aire a $60,2{ }^{\circ} \mathrm{C}$ y disminución de $R$, obteniendo Biot $A=B i_{R}$ y $B=D_{A B}$.

Tabla 5: Parámetros obtenidos de simulación por Lima (1999) y en la presente investigación.

\begin{tabular}{|c|c|c|c|c|c|c|c|c|c|}
\hline \multirow[b]{2}{*}{ Lima (1999) } & \multicolumn{6}{|c|}{ Modelo para un sólido esferoidal } & \multicolumn{3}{|c|}{ Modelo para un cilindro infinito } \\
\hline & Ensayo & $\begin{array}{c}\mathrm{T}_{\mathrm{a}} \\
\left({ }^{\circ} \mathrm{C}\right)\end{array}$ & $\begin{array}{c}\mathrm{D}_{\mathrm{AB}} \cdot 10^{10} \\
\left(\mathrm{~m}^{2} / \mathrm{s}\right)\end{array}$ & $\begin{array}{c}\mathrm{k}_{\mathrm{m}} \cdot 10^{8} \\
(\mathrm{~m} / \mathrm{s})\end{array}$ & $\begin{array}{c}\mathrm{Bi}_{\mathrm{L}} \\
\text { (adim.) }\end{array}$ & $\begin{array}{l}\alpha .10^{7} \\
\left(\mathrm{~m}^{2} / \mathrm{s}\right)\end{array}$ & $\begin{array}{c}\mathrm{D}_{\mathrm{AB}} \cdot 10^{10} \\
\left(\mathrm{~m}^{2} / \mathrm{s}\right)\end{array}$ & $\begin{array}{c}\mathrm{k}_{\mathrm{m}} \cdot 10^{8} \\
(\mathrm{~m} / \mathrm{s})\end{array}$ & $\begin{array}{c}\mathrm{Bi}_{\mathrm{L}} \\
\text { (adim.) }\end{array}$ \\
\hline \multirow{6}{*}{$\begin{array}{l}\text { Modelo II - } \\
\text { Condición de } \\
\text { contorno } \\
\text { convectiva }\end{array}$} & 1 & 29,9 & 6,02 & 6,10 & 5,78 & ---- & ---- & ---- & \\
\hline & 2 & 39,9 & 6,25 & 10,51 & 9,53 & ---- & ---- & ---- & \\
\hline & 3 & 49,9 & 13,27 & 15,43 & 6,63 & 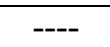 & ---- & ---- & \\
\hline & 4 & 60,2 & 25,87 & 17,03 & 3,75 & $\begin{array}{ll}--- \\
\end{array}$ & 23,83 & 23,83 & 5,70 \\
\hline & 5 & 60,5 & 25,90 & 19,38 & 4,27 & ---- & ---- & ---- & \\
\hline & 6 & 68,4 & 34,28 & 19,76 & 3,28 & ---- & ---- & ---- & \\
\hline \multirow{6}{*}{$\begin{array}{c}\text { Modelo III - } \\
\text { Condición de } \\
\text { contorno } \\
\text { convectiva + } \\
\text { disminución de } \\
\text { volumen }\end{array}$} & 1 & 29,9 & 1,65 & 10,10 & $\begin{array}{l}34,46 \\
18,40\end{array}$ & ---- & 1,35 & $\begin{array}{l}8,80 \\
8,80\end{array}$ & $\begin{array}{l}36,72 \\
19,61\end{array}$ \\
\hline & 2 & 39,9 & 2,48 & 15,53 & $\begin{array}{l}35,47 \\
21,38\end{array}$ & 1,20 & 2,32 & $\begin{array}{l}12,86 \\
12,86\end{array}$ & $\begin{array}{l}31,38 \\
18,91\end{array}$ \\
\hline & 3 & 49,9 & 4,57 & 21,35 & $\begin{array}{l}26,64 \\
15,75\end{array}$ & ---- & 4,01 & $\begin{array}{l}19,17 \\
19,17\end{array}$ & $\begin{array}{l}27,29 \\
16,14\end{array}$ \\
\hline & 4 & 60,2 & 7,25 & 22,30 & $\begin{array}{l}17,52 \\
11,14\end{array}$ & ---- & 6,95 & $\begin{array}{l}21,16 \\
21,16\end{array}$ & $\begin{array}{l}17,35 \\
11,03\end{array}$ \\
\hline & 5 & 60,5 & 7,30 & 26,15 & $\begin{array}{l}20,47 \\
12,89\end{array}$ & ---- & 6,71 & $\begin{array}{l}24,46 \\
24,46\end{array}$ & $\begin{array}{l}20,84 \\
13,12\end{array}$ \\
\hline & 6 & 68,4 & 8,63 & 26,56 & $\begin{array}{l}17,49 \\
11,23\end{array}$ & ---- & 9,69 & $\begin{array}{l}23,15 \\
23,15\end{array}$ & $\begin{array}{c}13,57 \\
8,71\end{array}$ \\
\hline \multirow{6}{*}{$\begin{array}{c}\text { Modelo III - } \\
\text { Condición de } \\
\text { contorno } \\
\text { convectiva + } \\
\text { disminución de } \\
\text { volumen + } \mathrm{k}_{\mathrm{m}} \\
\text { infinito }\end{array}$} & 1 & 29,9 & 0,93 & $\infty$ & --- & ---- & ---- & ---- & \\
\hline & 2 & 39,9 & 1,40 & $\infty$ & ---- & ---- & ---- & ---- & \\
\hline & 3 & 49,9 & 2,31 & $\infty$ & ---- & ---- & ---- & ---- & \\
\hline & 4 & 60,2 & 3,08 & $\infty$ & ---- & ---- & ---- & ---- & \\
\hline & 5 & 60,5 & 3,29 & $\infty$ & ---- & ---- & ---- & ---- & \\
\hline & 6 & 68,4 & 3,66 & $\infty$ & ---- & ---- & ---- & ---- & \\
\hline
\end{tabular}


Comparando las $\mathrm{D}_{\mathrm{AB}}$, presentados en la Tabla 4, del modelo (III) y las calculadas en este artículo por un modelo analítico, se obtiene una desviación promedio $X_{\text {prom }}=-6,1$ y desviación estándar $\sigma_{n-1}=10,3 \%$. Comparando los $\mathrm{k}_{\mathrm{m}}$, resultan en $\mathrm{X}_{\text {prom }}=-10,8 \mathrm{y} \sigma_{\mathrm{n}-1}=4,3$ y para los $\mathrm{Bi}_{\mathrm{m}}$, resultan en $\mathrm{X}_{\text {prom }}=-4$ y $\sigma_{\mathrm{n}-1}=$ 10,4 . Por lo tanto, la concordancia entre los parámetros del presente artículo y los del modelo (III), pueden ser considerados buenos; encontrándose en el rango de las desviaciones publicadas en la literatura, para determinaciones experimentales y teóricas de parámetros de transporte.

Tabla 6: Ecuaciones básicas de cálculo con el modelo analítico de cilindro infinito.

\begin{tabular}{|c|l|}
\hline Ensayo & \multicolumn{1}{c|}{ Ecuaciones básicas } \\
\hline 1 & $\frac{M_{A}-2,02 g}{48,43 g-2,02 g}=0,432953 \exp (-0,0004064 t)+0,7571252 \exp (-0,0312348 \mathrm{t})-0,2049494 \exp (0,0054482 \mathrm{t})$ \\
& $R=0,01613-0,0001717\left(48,43-M_{A}\right)$ \\
\hline 2 & $\frac{M_{A}-0,95 \mathrm{~g}}{45,16 \mathrm{~g}-0,95 \mathrm{~g}}=0,3936508 \exp (-0,061946 \mathrm{t})+0,5945779 \exp (-0,0238737 \mathrm{t})-0,0094498 \exp (0,0126561 \mathrm{t})$ \\
& $R=0,01569-0,0001541\left(45,16-M_{A}\right)$ \\
\hline 3 & $\frac{M_{A}-0,77 \mathrm{~g}}{42,79 \mathrm{~g}-0,77 \mathrm{~g}}=0,3329597 \exp (-0,0670232 \mathrm{t})+0,3526333 \exp (-0,0443582 \mathrm{t})+0,2958901 \exp (-0,081414 \mathrm{t})$ \\
\hline 4 & $\frac{R=0,01522-0,0001614\left(42,79-M_{A}\right)}{42,36 \mathrm{~g}-0,61 \mathrm{~g}}=1,5988845 \exp (-0,077787 \mathrm{t})-0,2887315 \exp (-0,0935546 \mathrm{t})-0,3137324 \exp (-0,0680589 \mathrm{t})$ \\
& $R=0,01530-0,0001436\left(42,36-M_{A}\right)$ \\
\hline 5 & $\frac{M_{A}-0,29 \mathrm{~g}}{41,40 \mathrm{~g}-0,29 \mathrm{~g}}=0,3335903 \exp (-0,0752869 \mathrm{t})+0,329089 \exp (-0,0700717 \mathrm{t})+0,3290852 \exp (-0,1283853 \mathrm{t})$ \\
& $R=0,01506-0,0001501\left(41,40-M_{A}\right)$ \\
\hline 6 & $\frac{M_{A}-0,18 \mathrm{~g}}{43,11 \mathrm{~g}-0,18 \mathrm{~g}}=0,3271191 \exp (-0,0910213 \mathrm{t})+0,5365081 \exp (-0,0936579 \mathrm{t})+0,1514727 \exp (-0,0909257 \mathrm{t})$ \\
& $R=0,01545-0,0001388\left(43,11-M_{A}\right)$ \\
\hline
\end{tabular}

\section{CONCLUSIONES}

Los valores de $k_{m}, D_{A B}$ y $B i_{L}$ obtenidos del modelo presentado en este trabajo, son del mismo orden de magnitud de los calculados por Lima (1999) en su modelo (III), usando métodos numéricos.

Los parámetros $\mathrm{k}_{\mathrm{m}}, \mathrm{D}_{\mathrm{AB}}, \mathrm{Bi}_{\mathrm{R}}$ y $\mathrm{Bi}_{\mathrm{L}}$ son obtenidos naturalmente del modelo desarrollado, sin necesidad de experimentos por separado para calcularlos ni de correlaciones para evaluarlos.

La disminución de volumen debe ser considerada en modelos de secado de frutas, pues se obtienen valores de $\mathrm{k}_{\mathrm{m}}, \mathrm{D}_{\mathrm{AB}}, \mathrm{Bi}_{\mathrm{R}}$ y $\mathrm{Bi}_{\mathrm{L}}$, que representan mejor el secado, en relación a los valores obtenidos con modelos de volumen constante.

\section{REFERENCIAS}

Abalone, R., A. Gastón y M.A. Lara; Simulación Numérica del Proceso de Secado de un Material Anisotrópico, Engenharia Térmica: 1, 47-55 (2001).

Bird, R.B., W.E. Stewart y E.N. Lightfoot; Transport Phenomena, John Wiley, New York, USA (2002).

Costa, A.R.S. y S.R. Ferreira; Sistema de Secado Solar para Frutos Tropicales, Información Tecnológica: 18(5), $49-58$ (2007). 
Costa, A.R.S.; Sistema de Secado Solar para Frutos Tropicales y Modelado del Secado de Banana en un Secador de Columna Estática, Tesis doctoral, UFRN - Universidad Federal de Río Grande del Norte, Natal, Brasil (2008). (En portugués y disponible en www.ufrn.br.)

Crank, J.; The Mathematics of Diffusion, Clarendon Press, Oxford, England (1976).

Karim, Md.A. y M.N.A. Hawlader; Drying Characteristics of Banana: Theoretical Modelling and Experimental Validation, Journal of Food Engineering: 70, 35-45 (2005a).

Karim, Md.A. y M.N.A. Hawlader; Mathematical Modelling and Experimental Investigation of Tropical Fruits Drying, International Journal of Heat and Mass Transfer: 48, 4914-4925 (2005b).

Kaya A., O. Aydın y I. Dincer; Experimental and Numerical Investigation of Heat and Mass Transfer During Drying of Hayward Kiwi Fruits (Actinidia Deliciosa Planch), Journal of Food Engineering: 88, 323330 (2008).

Lima, A.G.B., M.R. Queiroz y S.A. Nebra; Simultaneous Moisture Transport and Shrinkage During Drying of Solids with Ellipsoidal Configuration, Chemical Engineering Journal: 86, 85-93 (2002).

Lima, A.G.B.; Fenómeno de Difusión en Sólidos Esferoidales Prolatos. Estudio de Caso: Secado de Banana, Tesis doctoral, UNICamp - Universidad Estadual de Campinas, Facultad de Ingeniería Mecánica, Campinas, Brasil (1999). (En portugués y disponible en http://libdigi.unicamp.br/; el código del trabajo es vtls000188465.)

Luikov, A.V.; Analytical Heat Diffusion Theory. Academic Press, New York, USA (1968).

Luikov, A.V.; Heat and Mass Transfer in Capillary Porous Bodies, Pergamon Press, New York, USA (1966).

Mabrouk, S.B., B. Khiari y M. Sassi; Modelling of Heat and Mass Transfer in a Tunnel Dryer, Applied Thermal Engineering: 26, 2110-2118 (2006).

Mariani, V.C., A.G.B. Lima y L.S. Coelho; Apparent Thermal Diffusivity Estimation of the Banana During Drying Using Inverse Method, Journal of Food Engineering: 85, 569-579 (2008).

Pandey, R.N., S.K. Srivastava y M.D. Mikhailov; Solutions of Luikov Equations of Heat and Mass Transfer in Capillary Porous Bodies Through Matrix Calculus: a New Approach, International Journal of Heat and Mass Transfer: 42, 2649-2660 (1999).

Queiroz, M.R. y S.A. Nebra; Theoretical and Experimental Analysis of the Drying Kinetics of Bananas, Journal of Food Engineering: 47, 127-132 (2001).

Queiroz, M.R.; Estudio Teórico-experimental de la Cinética de Secado de Bananas, Tesis doctoral, UNICamp - Universidad Estadual de Campinas, Facultad de Ingeniería Mecánica (1994). (En portugués y disponible en http://libdigi.unicamp.br/; el código del trabajo es vtls000082363.)

Schwartzberg, H. G.; Mathematical Analysis of the Freezing and Thawing of Foods, AIChE Summer National Meeting, Detroit, Michigan (1981).

Silva, W.P. y C.M.D.P.S., Silva; LAB Fit Ajuste de Curvas (Regresión no Lineal y Tratamiento de Datos) v.7.2.14c (2003). (En portugués y disponible en http://www.angelfire.com/rnb/labfit/index_p.htm.)

Srikiatden, J. y J.S. Roberts; Predicting Moisture Profiles in Potato and Carrot During Convective Hot Air Drying Using Isothermally Measured Effective Diffusivity, Journal of Food Engineering: 84, 516-525 (2008). 
Teruel, B., L.A. Cortez, P. Leal y A.G.B Lima; Estudio Teórico del Enfriamiento de Frutas de Distintas Geometrías con Aire Forzado, Ciência e Tecnologia de Alimentos: 21(2), 228-235, mayo-agosto (2001). (En portugués.)

Wu, Y. y J. Irudayaraj; Analysis of Heat, Mass and Pressure Transfer in Starch Based Food Systems, Journal of Food Engineering: 29, 399-414 (1996). 